\title{
Helgason-Schiman Formula for Semisimple Lie Groups of Arbitrary Rank
} Bassey UN ${ }^{1 *}$ and Oyadare $\mathrm{OO}^{2}$

${ }^{1}$ Department of Mathematics, University of Ibadan, Ibadan, Nigeria

${ }^{2}$ Department of Mathematics, Obafemi Awolowo University, Ile-Ife, Nigeria

\begin{abstract}
This paper extends the Helgason-Schiffman formula for the $\mathrm{H}$-function on a semisimple Lie group of real rank one to cover a semisimple Lie group $G$ of arbitrary real rank. A set of analytic $\mathbb{R}$-valued cocycles are deduced for certain real rank one subgroups of $\mathrm{G}$. This allows a formula for the c-function on $\mathrm{G}$ to be worked out as an integral of a product of their resolutions on the summands in a direct-sum decomposition of the maximal abelian subspace of the Lie algebra $g$ of $\mathrm{G}$. Results about the principal series of representations of the real rank one subgroups are also obtained, among other things.
\end{abstract}

Keywords: Helgason-Schiffman formula; Spherical functions; Hfunction; Semi simple Lie group

\section{Introduction}

Let $\mathrm{G}$ be a semisimple Lie group with finite center and Lie algebra, $\mathrm{g}$. Define a Cartan involution on $\mathrm{G}$ as an involutive automorphism $\theta$ of $\mathrm{G}$ whose set of fixed points, $G^{\theta}=\{x \in G: \theta(x)=x\}$, is a maximal compact subgroup of G: We say $\mathrm{K}$ and $\theta$ are associated whenever $K=G^{\theta}$. In this case, set $\mathrm{t}=\{X \in \mathrm{g}: \theta X=X\}$ and $\mathrm{p}=\{\mathrm{X} \in \mathrm{g}: \theta \mathrm{X}=-\mathrm{X}\}$ Then $\mathrm{t}$ is the Lie algebra of $\mathrm{K}$ and we have the decompositions $\mathrm{g}=\mathrm{t} \oplus \mathrm{p}$ and $\mathrm{G}=\mathrm{K} \exp \mathrm{P}$ commonly called the Cartan decompositions of $g$ and G; respectively, associated to $\theta$. Now choose a maximal abelian subspace, a, of $\mathrm{p}$ and let $\mathrm{a}^{*}$ be its dual vector space. For any $\lambda \in a^{*}$ consider the subspace $\mathrm{g}_{\lambda}$ of $\mathrm{g}$ defined as $\mathrm{g}_{\lambda}=\{X \in \mathrm{g}:[H, X]=\lambda(H) X \forall H \in a\} . \lambda$ is called a root of the pair (g, a) whenever $\lambda \neq 0$ and $g_{\lambda} \neq\{0\}$. We therefore have the root-space decomposition, $\mathrm{g}=\mathrm{m} \oplus \oplus_{\lambda \in \Delta} g \lambda$, of $\mathrm{g}$; where $\mathrm{m}$ is the centralizer of $\mathrm{a}$ in $\mathrm{g}$ and $\Delta=\Delta(\mathrm{g}, \mathrm{a})$ denotes the set of all roots of $(\mathrm{g}$, a). $\mathrm{m}$ is $\theta$-stable and, hence, reductive in $\mathrm{g}$. If we set $\mathrm{m}_{1}=\mathrm{m} \cap \mathrm{t}$, then $\mathrm{m}=\mathrm{m}_{1} \oplus \mathrm{t}$.

Put a lexicographic ordering on $\mathrm{a}^{\star}$ and denote the subset of $\Delta$ consisting of positive roots of $(\mathrm{g}, \mathrm{a})$ as $\Delta^{+}$. Define $\mathrm{n}=\sum_{\lambda \in \Delta}+g_{\lambda}$ and $\mathrm{N}=\exp \mathrm{n}$. Then $\mathrm{n}$ is nilpotent subalgebra of $\mathrm{g}, \mathrm{N}$ is the closed analytic subgroup of $\mathrm{G}$ defined by $\mathrm{n}$, and $\exp (\mathrm{n} \rightarrow \mathrm{N})$ is an analytic diffeomorphism. We now have the Iwasawa decompositions $g=t \oplus a \oplus n$ and $\mathrm{G}=\mathrm{KAN}$ of $\mathrm{g}$ and G, respectively, with the abelian subgroup, A, defined as $A=\exp a$ : This decomposition of $\mathrm{G}$ gives rise to the projection maps $k: G \rightarrow K, a: G \rightarrow A, n: G \rightarrow N$, so that every $x \in G$ may be decomposed as $\mathrm{x}=\mathrm{K}(\mathrm{x}) \mathrm{a}(\mathrm{x}) \mathrm{n}(\mathrm{x})$. Since $a(x) \in A=\exp a$ we find that $\mathrm{a}(\mathrm{x})=\operatorname{expH} \mathrm{H}(\mathrm{x})$ where $H: G \rightarrow a$ is the composition of the maps $G \rightarrow A \rightarrow a$.The maps $\mathrm{K}, \mathrm{a}, \mathrm{n}$, and $\mathrm{H}$ are analytic maps on $\mathrm{G}$ and are known to contribute to many discussions of the harmonic analysis of $\mathrm{G}$. The $\mathbb{R}$ rank of $\mathrm{G}$; denoted as $\mathrm{m}$; is defined as the dimension of a. Since $I_{m}(H) \subseteq a$, it is therefore not unexpected that the analytic map $H: G \rightarrow a$ should have a relationship with the $\mathbb{R}$-rank of G: We refer to $H:=\log \circ a$ as the $\mathrm{H}$-function of $\mathrm{G}$.

For any G; with $\mathbb{R}$-rank one and Lie algebra g, there is an explicit expression for the $\mathrm{H}$-function which was independently established by Helgason and Schiffman [1]. Indeed the expression is completely defined on $\theta(\mathrm{N})$ and we have it as

$$
\lambda^{*}(H(\bar{n}))=\frac{1}{2} \log \left[\left(1+\frac{1}{2 d^{2}}|X|^{2}\right)^{2}+\frac{2}{d^{2}}|Y|^{2}\right]
$$

Where $\lambda^{*}$ is half of the only positive real root of $(g, a)$, $\bar{n}=\exp X \exp Y \in \theta(N),|X|:=-B(X, \theta X)$ and $\mathrm{B}$ is the Killing form on g, This may also be written as $e^{2 \lambda^{*}(\mathrm{H}(\bar{n}))}=\left(1+\frac{1}{2 d^{2}}|X|^{2}\right)^{2}+\frac{2}{d^{2}}|Y|^{2}$.

An analogous expression has been sought for other examples of $G$; starting in 1960 with the work of Bhanu-Murthy, whose study entails a group-by-group consideration, while the case of an arbitrary $\mathrm{G}$ is not known. A common feature of the computation of the $\mathrm{H}$-function for higher-than-one $\mathbb{R}$-rank groups, which is used to compute the $\mathrm{H}$-function on a group-by-group basis, is its relationship with the finite-dimensional representations of $G$. The above mentioned relationship is as follows: the $\mathrm{H}$-function of $\mathrm{G}$ relative to a minimal parabolic subgroup satisfies the relation $e^{2 \lambda^{*}(\mathrm{H}(\mathrm{x}))}=\left\|\Phi_{\lambda}(x) u\right\|^{2}$, where $\Phi_{\lambda}$ is a finite dimensional irreducible holomorphic representation of $G^{\mathbb{C}}$, simply connected group such that $G \subseteq G^{\mathbb{C}}$, with highest weight $\lambda$ and $\mathrm{u}$ is any unit vector in the sum of the weight spaces for weights that restricts to $\lambda$ on a [2].

We give the computation in the case of $G=S L(3, \mathbb{R})$. Let us write the subgroup $\bar{N}$ of $\mathrm{G}$ as $\bar{N}=\left\{\bar{n}:=\left(\begin{array}{lll}1 & 0 & 0 \\ x & 1 & 0 \\ z & y & 1\end{array}\right): x, y, z \in \mathbb{R}\right\}$. Then, from the above relation, it may be shown that $e^{2 \rho(H(\bar{n}))}=\left(1+x^{2}+z^{2}\right)\left(1+\mathrm{y}^{2}+(\mathrm{z}-\mathrm{xy})^{2}\right)$ for every $\bar{n} \in \bar{N}$. The $\mathrm{c}$-function in this case is then given as $c(\bar{n})=\iiint_{\mathbb{R}^{3}}\left(1+x^{2}+z^{2}\right)^{-\mathrm{a}}\left(1+\mathrm{y}^{2}+(\mathrm{z}-\mathrm{xy})^{2}\right)^{-\mathrm{b}} d x d y d z, a, b \in \mathbb{C}$, which, $\quad$ by $\quad$ an ingenious substitution becomes the product

$$
\iiint_{\mathbb{R}^{3}}\left(1+x^{2}\right)^{-\mathrm{a}}\left(1+\mathrm{y}^{2}\right)^{-\mathrm{b}}\left(1+z^{2}\right)^{-a-b+\frac{1}{2}} d x d y d z
$$

of three one-dimensional integrals. This is the Gindikin-Karpelevic

*Corresponding author: Bassey UN, Department of Mathematics, University of Ibadan, Ibadan, Nigeria, E-mail: unbassey@yahoo.com

Received July 21, 2014; Accepted November 29, 2014; Published December 05, 2014

Citation: Bassey UN, Oyadare OO (2014) Helgason-Schiman Formula for Semisimple Lie Groups of Arbitrary Rank. J Generalized Lie Theory Appl 9: 216. doi: 10.4172/1736-4337.1000216

Copyright: (c) 2014 Bassey UN, et al. This is an open-access article distributed under the terms of the Creative Commons Attribution License, which permits unrestricted use, distribution, and reproduction in any medium, provided the original author and source are credited. 
formula for $S L(3, \mathbb{R})$, which may be expressed in terms of gamma function. However, our interest here is to find the generalization of the expression for $e^{2 \rho(H(n))}$, that would work for every semisimple group $\mathrm{G}$ [3], In order to generalize the methods in the last paragraph to every semisimple Lie group $\mathrm{G}$ we seek the earlier mentioned relationship of $\mathrm{H}$ in terms of $m:=\mathbb{R}-\operatorname{rank}(\mathrm{G})$ : In this paper, we give an expression, in 2 for $\mathrm{H}$ which makes the harmonic analysis on $\mathrm{G} \mathbb{R}$-rank dependent. Indeed this expression leads to a generalization of the $\mathbb{R}$-rank one Helgason-Schiffman formula [1] to arbitrary rank as contained in 3 . This general formula reduces to the $\mathrm{H}$-function for $\mathrm{SL}(3, \mathbb{R})$, without using the method of the highest weight theorem for finite dimensional representations of $\mathrm{G}$.

\section{The Decomposition of the $\mathrm{H}$-function}

We start with Theorem 2.1 below which plays a fundamental role in what follows.

Theorem Let $\mathrm{G}$ be of $\mathbb{R}$-rank $\mathrm{m}$. Then we have

$$
H(x)=\sum_{j=1}^{m} t_{m, j}(x) \cdot X_{j}, x \in G
$$

Where $a=\operatorname{span}_{\mathbb{R}}\left\{X_{1}, \ldots, X_{m}\right\}$. In particular, each $x \mapsto t_{m, j}(x) \mathrm{a}$ logarithm function and is analytic on $\mathrm{G}$.

\section{Proof:}

The proof is essentially the same as in ([3], Theorem 2.1) and so is omitted

Before going on, we give the following notations which are required for what follows below. We know that the $\mathbb{R}-\operatorname{rank}(G)=m=\operatorname{dim}(a)$. For each $j \in\{1, \ldots, m\}$ choose a semisimple subalgebra $\mathrm{g}$; of $\mathrm{g}$ with a Cartan decomposition $g_{j}=t_{j} \oplus p_{j}$ such that $\{0\} \neq t, t_{j} \subset t$ and $p_{j} \subset p$. Fix a maximal abelian proper subspace aj of pj (assume throughout that aj is one-dimensional). Fix also a compatible order on non-zero restricted roots; here there are at most two roots which are positive with respect to this order, which we denote by $\alpha_{j}$ and $2 \alpha_{j}$ Thus, denoting by $\Delta_{j}=\Delta\left(g, a_{j}\right)$ the set of restricted roots of the pair $\left(\mathrm{g}_{\mathrm{j}}, \mathrm{a}_{\mathrm{j}}\right)$, then $\Delta_{j}=\left\{-2 \alpha_{j},-\alpha_{j}, \alpha_{j}, 2 \alpha_{j}\right\}$ with a corresponding positive system $\Delta_{j}^{+}=\left\{\alpha_{j}, 2 \alpha_{j}\right\}$ We denote by $\mu_{j}$ the linear functional on $\mathrm{a}_{j}$ which equals one half the largest positive restricted root of $\Delta_{j}$. We decompose a into a direct sum of one-dimensional m subspaces $a_{j}, 1 \leq j \leq m$, that is, $a=\oplus_{j=1}^{m} a_{j}$, with $\operatorname{dim}\left(a_{j}\right)=1$.

We employ the groups $S L(3, \mathbb{R})$ and $S p(2, \mathbb{R})$ to illustrate examples of the decomposition in the Theorem 2.1 above.

For the real rank 2 group $S L(3, \mathbb{R})$ a maximal abelian subspace, a, of $\mathrm{p}$ is

$$
a=\left\{\left(\begin{array}{ccc}
a_{1} & 0 & 0 \\
0 & a_{2} & 0 \\
0 & 0 & -\left(a_{1}+a_{2}\right)
\end{array}\right): a_{1}, a_{2} \in \mathbb{R}\right\} .
$$

We may then choose

$$
\left\{\left(\begin{array}{ccc}
a_{1} & 0 & 0 \\
0 & 0 & 0 \\
0 & 0 & -a_{1}
\end{array}\right): a_{1} \in \mathbb{R}\right\} \text { and }\left\{\left(\begin{array}{ccc}
0 & 0 & 0 \\
0 & a_{2} & 0 \\
0 & 0 & -a_{2}
\end{array}\right): a_{2} \in \mathbb{R}\right\}
$$

as $\mathrm{a}_{1}$ and $\mathrm{a}_{2}$, respectively, each of which is one-dimensional. In the case of $\mathrm{G}=$
$S p(2, \mathbb{R})$, a maximal abelian subspace is $\left\{\left(\begin{array}{cccc}s & 0 & 0 & 0 \\ 0 & t & 0 & 0 \\ 0 & 0 & -s & 0 \\ 0 & 0 & 0 & -t\end{array}\right): s, t \in \mathbb{R}\right\}$.
Thus $\left\{\left(\begin{array}{cccc}s & 0 & 0 & 0 \\ 0 & 0 & 0 & 0 \\ 0 & 0 & -s & 0 \\ 0 & 0 & 0 & 0\end{array}\right): s, \in \mathbb{R}\right\}$ and $\left\{\left(\begin{array}{llll}0 & 0 & 0 & 0 \\ 0 & t & 0 & 0 \\ 0 & 0 & 0 & 0 \\ 0 & 0 & 0 & -t\end{array}\right): t \in \mathbb{R}\right\}$ may

be chosen as a1 and a2; respectively.

It is clear that the case $m=1$ reduces to the situation of HelgasonSchiffmann. Next we discuss some of the properties of each of the maps $x \mapsto t_{m, j}(x)$. To this end let $a_{m, j}(x)=\exp \left(t_{m, j}(x) . X_{j}\right), x \in G, 1 \leq J \leq m$.

\section{Corollary}

We have $a(x)=\prod_{j=1}^{m} a_{m, j}(x), x \in G$.

This corollary generalizes an equivalent expression for $S L(m+1, \mathbb{R})$, established in [4] to any semisimple Lie group with finite center and of any real rank. One of the major applications of the $\mathrm{H}$-function, and now of Theorem 2.1, is its contribution to the compact picture of the induced representations on semisimple Lie groups. This contribution relies on the cocycle nature of $\mathrm{H}$. In anticipation of a similar use to be made of the maps $x \mapsto t_{m, j}(x)$ we establish the following proposition.

\section{Proposition}

Let there be given $j \in\{1, \ldots, m\}$. the map $x \mapsto t_{m, j}(x)$ induces an analytic $\mathbb{R}$-valued cocycle on $\mathrm{G}$.

\section{Proof}

Since $G / A N \simeq K$, the subgroup $\mathrm{K}$ may be regarded as a transitive homogeneous space for $\mathrm{G}$ acting from the left. We denote this action as $G \mathrm{XX} \rightarrow K:(x, k) \mapsto x[k]:=k(x k)$. In this context the function $x \mapsto a(x)$ induces an A-valued map $G \mathrm{XK} \rightarrow A:(x, k) \mapsto a(x: k)$ given simply as $a(x: k):=a(x k)$ and which satisfies

(i) $a(1: k)=1$

(ii) $a\left(x_{1} x_{2}: k\right)=a\left(x_{1}: x_{2}[k]\right) a\left(x_{2}: k\right)$, and

(iii) $a\left(x: x^{-1}[k]\right)=a\left(x^{-1} \mathrm{k}\right)^{-1}(c f .[7], p .84)$.

Now going over, from the map $(x, k) \mapsto a(x: k)$, to a (via the $\mathrm{H}$-function) and then to $\mathbb{R}$ (via each of $\mathrm{t}_{\mathrm{m}, \mathrm{j}}$ ), we may define the map $\left.(x, k) \mapsto \mu_{j}(\log \circ a)(x: k)\right)$, and denote it by $t_{m, j}(x: k)$. become

Using Theorem 2:1 above, properties (i), (ii) and (iii) of a (x : k)

(i)' $t_{m, j}(1: k)=0$

(ii)' $t_{m, j}\left(x_{1} x_{2}: k\right)=t_{m, j}\left(x_{1}: x_{2}[k]\right)+t_{m, j}\left(x_{2}: k\right)$, and

(iii)' $t_{m, j}\left(x: x^{-1}[k]\right)=-t_{m, j}\left(x^{-1} k\right)$.

The real rank 1 case of the last proposition is contained in Proposition 3.1 of [5]. It is known that the $\mathrm{H}$-function vanishes on the maximal compact subgroup $\mathrm{K}$. The implication is that each of the 
coefficient maps, $x \mapsto t_{m, j}(x)$, also vanish on $\mathrm{K}$.

The H-function is known to be completely defined on $\bar{N}=\theta(N)$, where $N=\exp (n), n=\oplus_{\alpha \in \Delta+(g, a)} g_{\alpha}$ and $\theta$ is the Cartan involution of $\mathrm{G}$ associated to $\mathrm{K}$. The decomposition of a in Theorem 2.1 means we consider the complete understanding of each of $t_{m, j}$ on the direct sum of eigenspaces corresponding to the positive restricted roots in $\Delta_{j}^{+}$. Hence a procedure for deriving an explicit expression for each of $t_{m, j}$ is to be accomplished on $\bar{N}_{j}=\theta\left(N_{j}\right)$, where $N_{j}=\exp \left(\mathrm{n}_{j}\right), \mathrm{n}_{\mathrm{j}}=\oplus_{\alpha \in \Delta_{j}^{+}} g_{\alpha}$. This, among other things, will be achieved in 3 below.

\section{The c-function and zonal Spherical Functions}

We now study the contributions of the decomposition of the $\mathrm{H}$-function in Theorem 2.1 to some aspects of harmonic analysis on G. These include the structure of spherical and c-functions and representations on G. Here we consider the c-function which appears as the coefficient-function of the eigenspace expansion of spherical functions.

Let $\rho$ be the half-sum of the positive roots of the pair ( $g$, a) with multiplicity. The $c$-function is given by the integral $c(\lambda)=\int_{\bar{N}} e^{-(\lambda+p) H(\bar{n})} d \bar{n}$, It is, however, customary to use the understanding of the function $j(\alpha)=\int_{-} e^{2 \alpha(H(\bar{n}))} d \bar{n}, \alpha \in \Delta^{+}$, in order to study the $\mathrm{c}$ function. Note that $c(\rho)=j(-\rho)$. We consider first the example of $S L(m+1, \mathbb{R})$

\section{Example}

$S L(m+1, \mathbb{R})$ : Take $\mathrm{m}=2$ for a start and introduce real parameters for members of $\bar{N}$ to have

$$
\begin{aligned}
& \bar{N}=\left\{\bar{n}=\left(\begin{array}{lll}
1 & 0 & 0 \\
x & 1 & 0 \\
z & y & 1
\end{array}\right): x, y, z \in \mathbb{R}\right\}, \\
& \text { With } a=\left\{\left(\begin{array}{ccc}
a_{1} & 0 & 0 \\
0 & a_{2} & 0 \\
0 & 0 & -\left(a_{1}+a_{2}\right)
\end{array}\right): a_{1}, a_{2} \in \mathbb{R}\right\} \text {. It is known [6] that }
\end{aligned}
$$

the $\mathrm{H}$-function, relative to a minimal parabolic subgroup $\mathrm{S}=\mathrm{MAN}$; is given by the relation $e^{2 \lambda(H(x))}=\left\|\Phi_{-}(x) u_{\lambda}\right\|^{2}$ where $\Phi_{\bar{\lambda}}$ is a finitedimensional irreducible holomorphic representations of $G^{\mathbb{C}}$, a simply connected group such that $G \subseteq G^{\mathbb{C}}$, with highest weight $\bar{\lambda}, \lambda=\left.\bar{\lambda}\right|_{a}, u_{\lambda}$ being any unit vector in the sum of the weight spaces for weights that restrict to $\lambda$ on $a$

The roots of the pair (g, a) are $\pm\left(e_{i}-e_{j}\right), 1 \leq i<j \leq 3$, Where

$$
e_{i}\left(\left(\begin{array}{ccc}
a_{1} & 0 & 0 \\
0 & a_{2} & 0 \\
0 & 0 & a_{3}
\end{array}\right)\right):=a_{i} .
$$

The corresponding positive system of restricted roots is $\Delta^{+}=\left\{\left(e_{1}-e_{2}\right),\left(e_{2}-e_{3}\right)\right\}$ on the requirements that $a_{1}>a_{2}, a_{2}>a_{3}, a_{1}>a_{3}$ [1]. It may be shown that $e^{2 e l(H(\bar{n}))}=1+x^{2}+z^{2}$ and $\quad e^{2(e l+e 2)(H(\bar{n}))}=1+y^{2}+(x y-z)^{2} . \quad$ Now since $\rho=e_{1}-e_{3}$ and $2 \rho=2 e_{1}-2 e_{3}=\left(2 e \rho_{1}\right)+2\left(e_{1}+e_{2}\right)$, then $e^{2 \rho(H(\bar{n}))}=\left(1+x^{2}+z^{2}\right)\left(1+y^{2}+(x y-z)^{2}\right)$ [6] Thus if we write complex numbers to describe the behaviour of $\lambda$ on $a$, then

$$
e^{-(\lambda+\rho)(H(\bar{n}))}=\left(1+x^{2}+z^{2}\right)^{-a}\left(1+y^{2}+(x y-z)^{2}\right)^{-b}, a, b \in \mathbb{C},
$$

and the c-function on $S L(3, \mathbb{R})$ is given as

$$
c(\lambda)=\int_{\bar{N}} e^{-(\lambda+\rho) H(\bar{n})} d \bar{n}=\int_{\mathbb{R}^{3}}\left(1+x^{2}+z^{2}\right)^{-a}\left(1+y^{2}+(x y-z)^{2}\right)^{-b} d x d y d z
$$

(since $\bar{N} \simeq \mathbb{R}^{3}$ ) We then have an expression for the $\mathrm{c}$-function on $S L(3, \mathbb{R})$ as the integral of complex indices of two polynomials.

The above situation may be generalised to the c-function on $S L(\mathrm{~m}+1, \mathbb{R})$. To this end we take $n$ to be a lower triangular matrix, $\left(x_{i j}\right)_{i, j=1}^{m+1}$, with 1 's on the diagonal. For each 1 with $1 \leq l \leq m$, a generalisation of the above computations is obtained by forming the sum of the squares of ${ }^{n} C_{l}$ minors of size l-by-l obtained from the first l columns of $\left(x_{i j}\right)_{i, j=1}^{m+1}$, The result is raised to a power depending on 1 , and the analogue of the c-function above is the integral over $\mathbb{R}^{\frac{1}{2} m(m+1)}$ of the product of $m$ expressions raised to their respective powers.

It is however known that the above construction techniques given for the c-function of $G=S L(m+1, \mathbb{R})$ do not extend to other real semisimple Lie groups with finite center. For this reason the earlier expression given as $e^{2 \lambda(H(x))}=\left\|\Phi_{-}(x) u_{\lambda}\right\|^{2}$ is always resorted to when ever the c-function of specific groups are needed, with the attendant restriction that there exists a simply connected group $G^{\mathbb{C}}$, such that $G \subseteq G^{\mathbb{C}}$ and with a_nite-dimensional irreducible holomorphic representation, $\Phi_{-}$. We give here an approach for the computation of the above $\mathrm{j}$-function (hence the $\mathrm{c}$-function) for any real rank $\mathrm{m}$ connected semisimple Lie group with finite center, which will establish the exact contribution of $m$ as earlier seen in the case of $S L(m+1, \mathbb{R})$.

\section{Theorem}

Let $\alpha_{j}=\left.\alpha\right|_{a j}$ and $\bar{N}_{j}=\bar{N}_{\alpha j} \cdot \bar{N}_{2 \alpha j}$ where every $n_{j} \in N_{j}$ is of the form $\quad \bar{n}_{j}=\exp Y_{j} \exp Z_{j}, Y_{j} \in g_{-\alpha j}, Z_{j} \in g_{-2 \alpha j}$. Introduce parameters that describe members of each $\bar{N}_{j}, 1 \leq j \leq m$, such that $\bar{N}=\bar{N}_{1} \ldots \bar{N}_{m}$. Then, for every $\alpha \in \Delta^{+}(g, a)$,

$$
j(\alpha)=\left\{\begin{array}{l}
\int_{g-\alpha j} \prod_{j=1}^{m}\left(1+\frac{1}{2} Q_{\alpha_{j}}\left(Y_{j}\right)\right)^{2} d Y_{j}, \quad \text { if each } 2 \alpha_{j} \notin \Delta_{j}, \\
\int_{g-\alpha j X \mathrm{X}-2 \alpha_{j}} \prod_{j=1}^{m}\left[\left(1+\frac{1}{2} Q_{\alpha_{j}}\left(Y_{j}\right)\right)^{2}+2 Q_{\alpha j}\left(Z_{j}\right)\right] d Y_{j} d Z_{j}, \quad \text { if each } 2 \alpha_{j} \in \Delta_{j},
\end{array}\right.
$$

Where $\alpha_{j}$ is chosen appropriately and $Q_{\alpha_{j}}$ is a quadratic form.

\section{Proof}

If $\alpha \in \Delta^{+}(g, a)$ then a choice may be made to have $\alpha_{j}=\left.\alpha\right|_{a j}>0$. Hence if $2 \alpha_{j} \in \Delta_{j}^{+}$, then $\alpha_{j}=\mu_{j}$, while if $2 \alpha_{j} \notin \Delta_{j}^{+}$, then $\alpha_{j}=2 \mu_{j}$, where is as defined under Theorem 2.1. Therefore 


$$
\begin{aligned}
e^{2 \alpha(H(\bar{n}))} & =e^{2 \alpha\left[\sum_{j=1}^{m} t_{m, j}(\bar{n}) \cdot X_{j}\right]} \\
& =\prod_{j=1}^{m} e^{2 \alpha j\left(t_{m, j}(\bar{n}) \cdot X_{j}\right)} \\
& = \begin{cases}\prod_{j=1}^{m}\left[e^{2 \mu j\left(t_{m, j}(\bar{n}) \cdot X_{j}\right)}\right]^{2}, & \text { if each } 2 \alpha_{j} \notin \Delta_{j}, \\
\prod_{j=1}^{m} e^{2 \mu j\left(t_{m, j}(\bar{n}) \cdot X_{j}\right)}, & \text { if each } 2 \alpha_{j} \in \Delta_{j} .\end{cases}
\end{aligned}
$$

Hence we restrict our computations to $e^{2 \mu j\left(t_{m, j}(\bar{n}) \cdot X_{j}\right)}$,

If we recall the definition of $\mu_{j}$ above, then

$$
\frac{1}{2} \mu_{j}=\left\{\begin{array}{ll}
\frac{1}{2}\left[\frac{1}{2}\left(\alpha_{j}\right)\right], & \text { if each } 2 \alpha_{j} \notin \Delta_{j}, \\
\frac{1}{2}\left[\frac{1}{2}\left(2 \alpha_{j}\right)\right], & \text { if each } 2 \alpha_{j} \in \Delta_{j} .
\end{array}= \begin{cases}\frac{1}{4} \alpha_{j}, & \text { if each } 2 \alpha_{j} \notin \Delta_{j} \\
\frac{1}{2} \alpha_{j}, & \text { if each } 2 \alpha_{j} \in \Delta_{j} .\end{cases}\right.
$$

each of which is not a root of the pair $\left(g_{j}, a_{j}\right)$. Hence $\mu_{j}$ is a short root of $\left(g_{j}, a_{j}\right)$ and we have the root-space decomposition $g_{j}=\left(m_{j} \oplus a_{j}\right) \oplus \sum_{\beta \in \Delta j} g_{\beta}$, where $m_{j} \oplus a_{j}$ is the centraliser of $a_{j}$ in $g\left(\mu_{j}\right):=g_{j}$. By construction $g\left(-\mu_{j}\right)=g\left(\mu_{j}\right)$, each $g\left(\mu_{j}\right)$ is stable under the restriction of the Cartan involution of $g$ and is therefore simple.

Denote by $G\left(\mu_{j}\right)$ the analytic subgroup of $\mathrm{G}$ corresponding to $g\left(\mu_{j}\right)$,while the $\mathrm{K}$ and $\mathrm{A}$ for $G\left(\mu_{j}\right)$ may be taken to be the connected groups $K\left(\mu_{j}\right)=K \cap G\left(\mu_{j}\right)$ and $A\left(\mu_{j}\right)=A \cap G\left(\mu_{j}\right)$ with $M\left(\mu_{j}\right)=M \cap K\left(\mu_{j}\right)$ as the corresponding M group. Thus the symmetric space $G\left(\mu_{j}\right) / K\left(\mu_{j}\right)$ has rank one, where each $G\left(\mu_{j}\right)$ is a real rank one semisimple Lie group with finite center. Hence we may define a quadratic form, $Q\left(\mu_{j}\right)$, as $Q_{\mu_{j}}(\mathrm{X})=\frac{4\langle X, \theta(X)\rangle}{\left\langle\bar{H}_{\mu j}, \theta\left(\bar{H}_{\mu j}\right)\right\rangle}, X \in g\left(\mu_{j}\right)$, where $\bar{H}_{\mu j}, \in a_{j}$ is such that $\mu_{j}\left(\bar{H}_{\mu_{j}}\right)=2$ and $\langle.,$.$\rangle is the restriction of$ the Killing form to $a_{j} \mathrm{X} a_{j}$.

It therefore follows that $e^{2 \alpha_{j}\left(t_{m, j}(\bar{n}) \cdot X_{j}\right)}$ is the $e^{2 \lambda(H(\bar{n}))}$ for the real rank one semisimple Lie group $G\left(\mu_{j}\right)$ (with $\mu_{j}$ given in terms of $\alpha_{j}$ as above). Hence

$$
\begin{aligned}
& e^{2 \alpha_{j}\left(t_{m, j}(\bar{n}) \cdot X_{j}\right)}=\left\{\begin{array}{l}
\left(1+\frac{1}{2} Q_{\alpha j}\left(Y_{j}\right)\right)^{2}, \\
\left(1+\frac{1}{2} Q_{\alpha j}\left(Y_{j}\right)\right)^{2}+2 \mathrm{Q}_{\alpha \mathrm{j}}\left(\mathrm{Z}_{\mathrm{j}}\right), \quad \text { if each } 2 \alpha_{j} \in \Delta_{j},
\end{array}\right. \\
& \text { required. }
\end{aligned}
$$

as required.

\section{Corollary}

Let $\alpha \in \Delta^{+}$Then the function $\bar{n} \mapsto e^{2 \alpha(H(n))}$ on $\bar{N}$ are polynomials in the Lie algebra coordinates on $n$

\section{Computation of $e^{\left.2 \alpha j\left(t_{m, j}(n)\right) \cdot X_{j}\right)}$ : the case of $\operatorname{SL}(3, \mathbb{R})$.}

We start by restricting the members of $\Delta^{+}=\left\{\left(e_{1}-e_{2}\right),\left(e_{2}-e_{3}\right) \cdot\left(e_{1}-e_{3}\right)\right\}$ to $a_{1}$ and $a_{2}$ to have

$\left(e_{1}-e_{2}\right)\left(\operatorname{diag}\left(a_{1}, 0,-a_{1}\right)\right)=a_{1},\left(e_{2}-e_{3}\right)\left(\operatorname{diag}\left(a_{1}, 0,-a_{1}\right)\right)=a_{1},\left(e_{1}-e_{3}\right)\left(\operatorname{diag}\left(a_{1}, 0,-a_{1}\right)\right)=$

$2 a_{1}$ for $a_{1}$, and $\left(e_{1}-e_{2}\right)\left(\operatorname{diag}\left(0, a_{2},-a_{2}\right)\right)=-a_{2},\left(e_{2}-e_{3}\right)\left(\operatorname{diag}\left(0, a_{2},-a_{2}\right)\right)=$

$2 a_{2},\left(e_{1}-e_{3}\right)\left(\operatorname{diag}\left(0, a_{2},-a_{2}\right)\right)=a_{2}$ for $a_{2}$
If we now require, in addition to the earlier requirements of Example 3.1, that $a_{1}>0$ and $a_{2}>0$, we may define $\alpha_{1}: a_{1} \rightarrow \mathbb{R}$ and $\alpha_{2}: a_{2} \rightarrow \mathbb{R}$ as $\alpha_{1}\left(H_{1}\right)=a_{1}, \mathrm{H}_{1} \in a_{1}$ and $\alpha_{2}\left(H_{2}\right)=a_{2}, \mathrm{H}_{2} \in a_{2}$, respectively. These are respectively the restrictions $\left.\left(e_{1}-e_{2}\right)\right|_{a_{1}}$ and $\left.\left(e_{1}-e_{3}\right)\right|_{a_{2}}$, with $2_{\alpha_{1}}=\left.\left(e_{1}-e_{3}\right)\right|_{a_{1}}$, and $2_{\alpha_{2}}=\left.\left(e_{2}-e_{3}\right)\right|_{a_{2}}$.

If we then define $g\left(\alpha_{1}\right)=a_{1} \oplus g_{\alpha_{1}} \oplus g_{2 \alpha_{1}} \oplus g_{-\alpha_{1}} \oplus g_{-2 \alpha_{1}}$ and $g\left(\alpha_{2}\right)=a_{2} \oplus g_{\alpha_{2}} \oplus g_{2 \alpha_{2}} \oplus g_{-\alpha_{2}} \oplus g_{-2 \alpha_{2}} \quad$ (since $\quad \mathrm{m}=0$ ), then $t(j)=g\left(\alpha_{j}\right) \cap t$ and $\mathrm{p}(j)=g\left(\alpha_{j}\right) \cap p$, with $\mathrm{N}_{j}=\exp \left(g_{\alpha_{j}} \oplus g_{2 \alpha_{j}}\right)$. The restriction of members of $\Delta^{+}$to $a_{j}$ shows that $2 \alpha_{j} \in \Delta_{j}^{+}$and we may conclude that each $g\left(\alpha_{j}\right)$ is isomorphic with a real rank one (semi-) simple Lie algebra with $\Delta_{j}=\left\{ \pm \alpha_{j}, \pm 2 \alpha_{j}\right\}$, so that

$$
e^{2 \alpha_{j}\left(t_{2, j}\right)(\bar{n}) \cdot X_{j}}=\left(1+\frac{1}{2} Q_{\alpha_{j}}\left(Y_{j}\right)\right)^{2}+2 Q_{\alpha_{j}}\left(Z_{j}\right)
$$

For $\bar{n}=\exp \left(Y_{j}+Z_{j}\right), 1 \leq \mathrm{j} \leq 2$. This is as computed earlier in Example 3.1.

Another approach to the construction of $g\left(\mu_{j}\right)$ is as follows. Let $\mathrm{m}_{j}^{1}$ be the centraliser of $a_{j}$ in $\mathrm{g}$. It may be shown that $\mathrm{m}_{j}^{1}$ is stable under the restriction of the Cartan involution and that the analytic subgroup, $M_{j}^{1}$, of G corresponding to $\mathrm{m}_{j}^{1}$, is the centraliser of $a_{j}$ in $\mathrm{G}$. We set $m_{j}=m_{j}^{1} \cap t$ and $M\left(\mu_{j}\right)=M_{j}^{1} \cap K$.

Let us now choose $a$ to be a short root of the pair (g,a), i.e., $\alpha \in \Delta^{+}$such that $\frac{1}{2} \alpha \notin \Delta$. We may choose $\alpha_{j}$ by restrictions as in Computation 3:4 and compute the algebra

$$
g_{\alpha_{j}}=\left\{X \in g: \operatorname{ad}(H) X=\alpha_{j}(H) X, \forall H \in a_{j}\right\},
$$

from which we now define $g\left(\alpha_{j}\right)=m_{j} \oplus a_{j} \oplus g_{\alpha_{j}} \oplus g_{2 \alpha_{j}} \oplus g_{-\alpha_{j}} \oplus g_{-2 \alpha_{j}}$.

We are now in a position to employ Proposition 2:3 to construct the compact picture of the induced representation on $G\left(\mu_{j}\right)$. Fix $j \in\{1, \ldots, m\}$. Let $A_{j}=\exp \left(a_{j}\right), \lambda_{j} \in\left(a_{j}^{*}\right)^{\mathbb{C}}=a_{j}^{*}+i a_{j}^{*} \quad$ and define $\xi_{\lambda_{j}}: A_{j} \rightarrow \mathbb{C}^{*}:=\mathbb{C} \backslash\{0\}$ by the requirement $\xi_{\lambda_{j}}(\mathrm{a})=e^{\lambda_{j}(\log a)} . \xi_{\lambda_{j}}$ is a quasi-character of $A_{j}$ and is unitary iff $\lambda_{j} \in i a_{j}^{*}$ We therefore have the following.

\section{Proposition}

The map $(x, k) \mapsto \xi_{\lambda_{j}}\left(a_{m, j}(x: k)\right)$, for $x \in G\left(\mu_{j}\right), k \in K\left(\mu_{j}\right)$, is an analytic $\mathbb{C}^{*}$-valued cocycle.

\section{Proof}

By Proposition 2.3

Setting $\rho_{j}=\frac{1}{2} \sum_{\beta \in \Delta_{j}^{+}} \operatorname{dim}\left(g_{\beta}\right)$. $\beta$, we define $\pi_{\sigma_{j}, \lambda_{j}}$, as

$\left(\pi_{\sigma_{j}, \lambda_{j}}(x) f\right)(\mathrm{k})=\mathrm{e}^{-\left(\lambda_{\mathrm{j}}+\rho_{\mathrm{j}}\right)\left(\mathrm{t}_{\mathrm{m}, \mathrm{j}}\left(x^{-1} k\right) \cdot X_{j}\right)} f\left(x^{-1}[K]\right)$,

$x \in G\left(\mu_{j}\right), k \in K\left(\mu_{j}\right)$, with $f \in h\left(\sigma_{j}\right)$, where

$h\left(\sigma_{j}\right):=\left\{g \in L^{2}\left(K\left(\mu_{j}\right)\right): g(x m)=\sigma_{j}(m)^{-1} g(x), m \in M\left(\mu_{j}\right) \cap \mathrm{K}\left(\mu_{j}\right), x \in G\left(\mu_{j}\right)\right\}$,

$\sigma_{j}$ a finite-dimensional unitary representation on $M\left(\mu_{j}\right)$ Details of the construction of $\pi_{\sigma_{j}, \lambda_{j}}$ may be found in [5].

\section{Proposition}

$\pi_{\sigma_{j}, \lambda_{j}}$ is an irreducible unitary representation of $G\left(\mu_{j}\right)$ on 
$h\left(\sigma_{j}\right)$, for $\lambda_{j} \in i a_{j}^{*}$ and irreducible $\sigma_{j}$ It reduces to the left-regular representation on $\Upsilon_{j}:=\left\{x \in G\left(\mu_{j}\right): t_{m, j}(x: k)=0, \forall k \in K\right\}$.

\section{Proof}

The cocycle relations proved in Proposition 2.3 for $t_{m, j}$ give $\pi_{\sigma_{j}, \lambda_{j}}(1)=1$ and $\sigma_{\sigma_{j}, \lambda_{j}}(x y)=\pi_{\sigma_{j}, \lambda_{j}}(x) \pi_{\sigma_{j}, \lambda_{j}}(y), \forall x, y \in G\left(\mu_{j}\right)$ while the continuity of the map $(x, f) \mapsto \pi_{\sigma_{j}, \lambda_{j}}(x) f$ of $G\left(\mu_{j}\right) \times h\left(\sigma_{j}\right)$ into $h\left(\sigma_{j}\right)$ the irreducibility and unitarity of $\pi_{\sigma_{j}, \lambda_{j}}(x)$ are established exactly as in the case of the principal series on G.

If $x \in \Upsilon_{j}$, then from the same cocycle properties of $t_{m, j}$, we have that $x^{-1} \in \Upsilon_{j}$ Thus $t_{m, j}\left(x^{-1} k\right)=t_{m, j}\left(x^{-1}: k\right)=0$.

It is known that each of the real rank one semisimple Lie groups, $G\left(\mu_{j}\right)$ admits the induced representations, $I n d_{\Upsilon_{j}}^{G(\mu j)}$ which may be restricted to $K\left(\mu_{\mathrm{j}}\right)$ to get all the principal series of representations of $G\left(\mu_{j}\right)$. In this light a consequence of the above Proposition is the following.

\section{Corollary}

Let $\sigma_{j}$ be a finite-dimensional irreducible unitary representation of $M\left(\mu_{j}\right)$ and $\lambda_{j} \in i a_{j}^{*}$. The representations $\pi_{\sigma_{j}, \lambda_{j}}$ exhausts the unitary principal series of $G\left(\mu_{j}\right)$.

We are now encouraged to define the spherical functions $x \mapsto \varphi_{\lambda_{j}}(x), x \in G\left(\mu_{j}\right)$ corresponding to the class 1 members of $\pi_{\sigma_{j}, \lambda_{j}}$ With respect to the spherical function, $\varphi_{\lambda}(x)=\int_{K} e^{-(\lambda+\rho)\left(H\left(x^{-1} k\right)\right)} d k$ of $\mathrm{G}$, we refer to $\varphi_{\lambda_{j}}$ as the resolution of the spherical function $\varphi_{\lambda}$.

The Plancherd measure $\mu$ is supported on the set of real-valued $\lambda$ and is of the form

$$
d \mu\left(\varphi_{\lambda}\right)=\text { const } \cdot \frac{d \lambda}{|c(\lambda)|^{2}}
$$

Where $d \lambda$ is the Lebesgue measure on the dual of the real vector space a and the function $c$ is given explicitly as a product of betafunctions by the following formula,

$$
c(\lambda)=\prod_{a \geq 0} B\left(\frac{1}{2} m_{a}, \frac{1}{4} m_{\frac{a}{2}}+\frac{1}{2} i \lambda\left(a^{v}\right)\right)
$$

where the product is over the positive roots relative to some ordering, $m_{a}$ is the multiplicity of the root a, and $a^{v} \in a$ is the dual root corresponding to $\mathrm{a}$, that is,

$$
\lambda\left(a^{v}\right)=\frac{2\langle\lambda, a\rangle}{\langle a, a\rangle}
$$

The explicit calculation (3.1) of $c(\lambda)$ is due to Bhanu - Murthy [7] for the split groups and to Gindikin and Karpelevic in the general case [1].

We define a representation $\pi$ on a (locally convex) space $\mathrm{V}$ to be of class-1 whenever the subspace $V^{K}:=\{v \in V<: \pi(k) v=v, k \in K\}$ of all $\mathrm{K}$-invariant vectors in $\mathrm{V}$, is of dimension 1 . It is known [8] that class-1 representations are associated with spherical functions on $G$ (which are the matrix coefficients of these representations), and that, for irreducible $\sigma$, the (unitary) principal series, $\pi_{\sigma, \lambda}$ is of class- 1 if, and only if, $\sigma$ is the trivial representation on $\mathrm{M}$. Let us therefore denote $\pi \lambda:=\pi_{1, \lambda}$ and set the matrix coefficient of $\pi_{\lambda}$ defined by the function 1 , as $\varphi_{\lambda}$ given as

$$
\varphi_{\lambda}(x)=\left(\pi_{\lambda}(x) 1,1\right)
$$

Where $x \in G, \lambda \in F=a_{c}^{*}, 1 \in L^{2}(K)$ and (...) is an inner product on $\mathrm{L}^{2}(\mathrm{~K})$. The Function $\varphi_{\lambda}$ is spherical and, has the integral representation $\varphi_{\lambda}(x)=\int_{k} e^{-(\lambda+\rho)\left(H\left(x^{-1} k\right)\right)} d k$ as given above.

The result of Theorem 3.2 leads to the following product formula for the spherical functions, $\varphi_{\lambda}$, in a direction different from the Gindinkin-Karpelevic product formula for spherical functions.

\section{Theorem}

Every spherical function, $\varphi_{\lambda}, \lambda \in F$, on $\mathrm{G}$ is of the form

$$
\varphi_{\lambda}(x)=\prod_{j=1}^{m} \varphi_{\lambda_{j}}(x)
$$

where each $\varphi_{\lambda j}(x)$ is the resolution of $\varphi_{\lambda}(x)$ on each summand in the direct sum $\oplus_{j=1}^{m} a_{j}=a$.

\section{Proof}

We first note that

$$
\begin{aligned}
& \left(\pi_{\lambda}(x) f\right)(k)=\left(\xi_{\lambda} \cdot \delta\right)\left(a\left(x^{-1} k\right)\right)^{-1} f\left(x^{-1}[k]\right) \\
& =e^{-\lambda \log \left(a\left(x^{-1} k\right)\right)} \cdot e^{\left.-\rho \log \left(a x^{-1} k\right)\right)} f\left(x^{-1}[k]\right) \\
& =e^{-(\lambda+\rho) \log \left(a\left(x^{-1} k\right)\right)} f\left(x^{-1}[k]\right) \\
& =e^{\left.-(\lambda+\rho)\left(H x^{-1} k\right)\right)} f\left(x^{-1}[k]\right) \\
& =\mathrm{e}^{-(\lambda+\rho)\left(\sum_{j=1}^{m}\left(t_{j}\left(x^{-1} k\right) H_{j}\right)\right)} f\left(x^{-1}[k]\right) \\
& =\prod_{j=1}^{m} e^{-(\lambda+\rho)\left(t_{j}\left(x^{-1} k\right) H_{j}\right)} f\left(x^{-1}[k]\right)
\end{aligned}
$$

which is substituted into $\varphi_{\lambda}(x)=\left(\pi_{\lambda}(x) 1,1\right)$ gives

$$
\varphi \lambda \mathrm{x}=\prod_{j=1}^{m}\left(\int_{K} e^{-(\lambda+\rho)\left(t j\left(x^{-1} k\right) H j\right)} d k\right)
$$

The expression $\left.\int_{K} e-(\lambda+\rho)\left(t_{j}\left(x^{-1} k\right) H_{j}\right)\right) d k$ is the resolution of $\varphi_{\lambda}(x)$ on each aj and is denoted

As $\varphi_{\lambda_{j}}(x)$.

The product formula above explains that spherical functions, $\varphi_{\lambda}(x)$ on any real rank $\mathrm{m}$ group $\mathrm{G}$, is the product of its resolutions, $\varphi_{2}(x)$ on each of the 1-dimensional subspaces, aj of a. It implies that spherical functions on real rank $m$ groups can be studied through its resolutions, on some 1-dimensional subspace.

\section{References}

1. Warner G (1972) Harmonic analysis on semisimple Lie groups II. Grundlehren dermathematischen Wissenschaften 189.

2. Knapp AW (1986) Representation theory of semisimple groups, an overview based onexamples. Princeton University Press, Princeton.

3. Bassey UN, Oyadare OO (2013) A Theorem on the Iwasawa Projection and Applications to Some Representations of Reductive Groups. Universal J Math Math Sci 3: 157-164.

4. Varadarajan VS (1999) An introduction to harmonic analysis on semisimple Lie groups. Cambridge University Press .

5. Bassey UN, Oyadare OO (2009) Remarks on the principal series of representations: the case of SL(2, $\mathbb{R})$. JP J Geometry Topology 9: 249-262.

6. Knapp AW (2002) Lie groups beyond an introduction. Progress in Mathematics 140. 
Citation: Bassey UN, Oyadare OO (2014) Helgason-Schiman Formula for Semisimple Lie Groups of Arbitrary Rank. J Generalized Lie Theory Appl 9: 216. doi: 10.4172/1736-4337.1000216

Page 6 of 6

7. Bhanu MTS (1960) The asymptotic behaviour of zonal spherical functions on the Siegel upper-half plane. Doclady Akad Nauk SSSR 135: 1025-1029.
8. Gangolli R, Varadarajan VS (1988) Harmonic analysis of spherical functions on real reductive groups. Ergebnisse der Mathematik und ihrer Grenzgebiete 101 